\title{
Erratum: Phonon Transmission at a Liquid Helium-Solid Interface: The Influence of Surface Roughness and Finite Lifetimes of the He States*
}

\author{
R. Haug, E. Sigmund, and K. Weiss $\dagger$ \\ Institut für Theoretische Physik, Universität Stuttgart, Stuttgart, West Germany
}

(Received September 5, 1987)

The section from Eq. (32) to Eq. (33) inclusive, appearing in the middle of p. 36 , should be placed after the last line of that page.

*This paper appeared in J. Low Temp. Phys. 67, 27 (1987).

†HILTI AG, FL-9494 Schaan, Liechtenstein. 